\title{
Revisão Sistemática de Estudos sobre Programas de Treinamento Parental
}

\author{
Ariane Bochi ${ }^{1}$ \\ Daiana Friedrich \\ Universidade Federal de Ciências da Saúde de Porto Alegre, Porto Alegre, RS, Brasil \\ Janaína Thais Barbosa Pacheco \\ Departamento de Psicologia da Universidade Federal de Ciências da Saúde de Porto Alegre, \\ Porto Alegre, RS, Brasil
}

\begin{abstract}
Resumo
O treinamento parental é uma prática que visa sistematizar o repertório comportamental emitido pelos pais quanto ao manejo do comportamento dos filhos, inserindo-se como estratégia frequentemente utilizada nas questões de relacionamento familiar. Através de uma revisão sistemática da literatura nacional e internacional, o presente artigo analisou as características dos estudos empíricos sobre treinamento de pais de crianças e/ou adolescentes, realizados em grupo. Foram analisados 27 artigos publicados entre 2006 e junho de 2014, indexados nas bases Literatura Latino-Americana e do Caribe em Ciências da Saúde (Lilacs), Scientific Electronic Library Online (SciELO), Medline e PsycInfo. Na análise dos dados, foram enfocadas as seguintes categorias: título do artigo, autores, ano de publicação, objetivo do estudo, público alvo, tamanho da amostra, delineamento, estratégias de intervenção e principais resultados. Os resultados apontam que o delineamento mais utilizado foi o ensaio clínico randomizado. Além disso, a maioria das intervenções teve foco nos pais, ou nos pais e crianças. Verifica-se que o maior número das técnicas utilizadas no treinamento de pais foram técnicas cognitivo-comportamentais. Este artigo discute limitações e contribuições para a área.
\end{abstract}

Palavras-chave: Treinamento parental, pais, intervenção em grupo.

\section{A Systematic Review of Studies on Parental Training Programs}

\begin{abstract}
Parent training is a practice that aims to organize the behaviour repertory issued by the parents when dealing with their children behaviour, becoming a frequent strategy used to work with family relationship issues. Trough a systematic review of the national and international literature, this article analyzed the methodological features of empirical studies about training for children and/or teenagers parents, conducted in group. 27 articles were analyzed, published between 2006 and July 2014, indexed in the Literatura Latino-Americana e do Caribe em Ciências da Saúde (Lilacs), Scientific Electronic Library Online (SciELO), Medline and PsycInfo bases. In the data analysis, the following categories were focused: article title, author, publishing date, study objective, audience, sample size, delineation, strategies of intervention and the main results. The results indicated that the most used design was the randomized clinical trial. Furthermore, most of the interventions were focused on parents or parents
\end{abstract}

Endereço para correspondência: Universidade Federal de Ciências da Saúde de Porto Alegre, Departamento do Curso de Psicologia, Rua Sarmento Leite, 245, Sala 208, Centro Histórico, Porto Alegre, RS, Brasil 90050170. E-mail: arianebochi@hotmail.com,daiana_friedrich@hotmail.com e janainapacheco@uol.com.br 
and their children. In addition, it can be observed that the cognitive-behavioural techniques were the most utilized techniques for the parents training. The present article discusses the limitations and the contribution for this field of study.

Keywords: Parent training, parents, intervention in group.

\section{Revisión Sistemática de Estudios sobre los Programas de Capacitación de los Padres}

\section{Resumen}

La capacitación de los padres es una técnica que tiene como meta coordinar el repertório de conductas de los padres en relacion con el comportamiento de sus hijos, insertada como una estratégia frecuentemente utilizada para trabajaren los problemas de relación familiar. Trás una revisión sistemática de la literatura nacional e internacional, este artículo intenciona el análize de las características metodológicas de los estudios empíricos sobre la capacitación de los padres de niños y/o adolescentes, desarrollados en grupo. Se analizaron 27 artículos publicados entre 2006 y junio de 2014, indexadas en las plataformas Literatura Latino-Americana e do Caribe em Ciências da Saúde (Lilacs), Scientific Electronic Library Online (SciELO), Medline y PsycInfo. En el análisis de datos se centraron las categorias seguientes: título del artículo, los autores, año de publicación, la meta del estudio, público alvo, tamaño de la muestra, límites, estratégias de intervención y resultados principales. Los resultados muestran que el diseño más ampliamente usado fue un ensayo clínico aleatorizado. Además, la mayoría de las intervenciones se habían centrado en los padres, o en los padres y hijos. Se verificó que el mayor número de técnicas que se utilizan en la capacitación de los padres eran técnicas cognitivo-comportamentales. Este artículo analiza las limitaciones y contribuciones para el assunto.

Palabras clave: Capacitación de los padres, padres, intervención en grupo.

Há algumas décadas vêm-se discutindo a importância da estrutura familiar e do estilo de criação para o desenvolvimento de crianças e adolescentes (Loeber \& Hay, 1997; Patterson, 1986). Tais fatores inspiraram a elaboração de programas específicos, que têm sido aplicados com diversos objetivos, dentre eles, o de capacitar os pais em suas dificuldades com crianças que apresentam problemas de comportamento (Kazdin, 1997; McMahon, 1999; Webster-Stratton, 1998), dificuldades escolares (Coser, Cortegoso, \& Gil, 2011), problemas alimentares (Boutelle et al., 2011, Haywood \& McCann, 2009; Lochrie et al., 2013) e sintomas depressivos (Wong, Gonzales, Montaño, Dumka, \& Millsap, 2014).

Patias, Siqueira e Dias (2013), apoiados em estudos que demonstraram a influência das práticas educativas como fatores de risco e de proteção, ressaltam a relevância da realização de programas de intervenção junto aos cuidadores, para orientá-los sobre as consequências de tais estratégias. Visto que muitos desses pais não possuem conhecimento sobre como melhorar suas formas de educar, torna-se fundamental investir em orientação para que possam aprimorá-las, reduzindo o risco de desenvolvimento de psicopatologias em crianças e adolescentes.

Dentro do contexto clínico e da saúde, o treinamento de pais é uma prática que procura sistematizar o repertório comportamental emitido por pais e mães em relação ao manejo da conduta de seus filhos com base no princípio da educação assertiva, na medida em que abrange formas de interação não coercivas e pró-sociais (Bolsoni-Silva \& Marturano, 2002). Nesse âmbito, o treinamento para pais insere-se como estratégia bastante utilizada para trabalhar questões de relacionamento e habilitar pais para alcançar melhores resultados em relação à educação de seus filhos (Bolsoni-Silva, Silveira, \& Marturano, 2008; Edwards, Sullivan, Meany-Walen, \& 
Kantor, 2010; Forehand et al., 2012; Marinho \& Silvares, 2001; McGilloway et al., 2012; Pardo \& Carvalho, 2011).

Os modelos de treinamento para pais são descritos na literatura especializada como uma das possibilidades de intervenção psicoterapêtica para a família (Donohue et al., 2014; Hodgetts, Savage, \& McConnell, 2013; Kazdin, 2003). Para alguns transtornos, esse modelo apresenta maior eficácia do que intervenções desenvolvidas apenas com crianças, uma vez que o ambiente familiar e social produz grande impacto no desenvolvimento infantil e prejuízos nas relações familiares podem ser responsáveis pela gênese de problemas de comportamento em crianças (Velasquez, Souza, Adjuto, Muñoz, \& Silveira, 2010).

Estudos têm demonstrado que o treinamento de pais proporciona resultados positivos abrangentes, envolvendo diferentes habilidades e voltados para problemas diversos, tais como: ampliação do repertório de habilidades sociais educativas e maior uso de reforçamento positivo dos pais (Jouriles et al., 2010; Reed et al., 2013), diminuição dos problemas de comportamento (Bagner, 2013; McGilloway et al., 2012; Smith, Dishion, Shaw, \& Wilson, 2013), melhora no desempenho acadêmico das crianças (Brennan et al., 2013; Spoth, Randall, \& Shin, 2008), e déficit de atenção e hiperatividade (ChronisTuscano et al., 2013; Hauth-Charlier \& Clément, 2014; Pfiffner, Villodas, Kaiser, Rooney, \& McBurnett, 2013).

Em pesquisa realizada por Caleiro e Silva (2012) verificou-se que as intervenções responsáveis por maior efetividade do treinamento de pais são aquelas que têm por base orientar os mesmos a desenvolverem uma relação mais afetuosa com seus filhos e a reforçarem positivamente o comportamento adequado das crianças. Estilos parentais altamente exigentes e punitivos são diretamente relacionados a problemas de comportamento na infância.

A avaliação da eficácia dos treinamentos de pais e o desenvolvimento de protocolos estruturados de intervenção constituem-se em desafios metodológicos aos pesquisadores. Bolsoni-Silva, Silveira e Ribeiro (2008) avaliaram os resultados de um programa de intervenção realizado com pais que apresentavam dificuldades de interação social com os filhos. Revelou-se o aumento do repertório de habilidades sociais-educativas das mães, com resultados positivos em habilidades como comunicação, expressividade e estabelecimento de limites. Consequentemente, tais mudanças resultaram na redução de problemas de comportamento e no acréscimo de respostas socialmente habilidosas das crianças.

Nessa direção, o estudo de Weber, Brandenburg e Salvador (2006) verificou a eficácia do programa "Promoção da Qualidade de Interação Familiar" (PQIF), um protocolo estruturado de intervenção que orienta e capacita pais para que eles mesmos aprendam a manejar as contingências de práticas educativas. A análise qualitativa dos relatos dos pais demonstrou que os participantes passaram por um processo de autoconhecimento e apresentaram mudanças, como: aumento da participação e do envolvimento dos pais na vida dos filhos, estabelecimento de regras claras e consistentes, maior valorização de comportamentos adequados dos filhos e aumento da freqüência de elogios por parte dos pais, além da diminuição ou abandono do uso de palmadas. Ademais, o alto índice de participação dos pais $(87,9 \%)$ foi considerado um indício positivo a favor da eficiência do programa.

Além disso, pesquisas têm sido realizadas na intenção de adaptar culturalmente programas de intervenção, como é o caso do estudo de Turner e Sanders (2007), que objetivou avaliar a adaptação do Programa de Parentalidade "Group Triple P - Positive Parenting Program" quanto à aceitabilidade e eficácia em uma comunidade indígena Australiana. O estudo de Lee, Griffiths, Glossop e Eapen (2010), mostrou sucesso com a mesma população ao testar o "Programa de $\mathrm{Pa}$ rentalidade Boomerangs", que possui uma base clínica e objetiva promover o aprimoramento da relação pai-filho. Neste último, apesar da pequena amostra não ter permitido a generalização dos resultados para a população em questão, as mães participantes forneceram feedback positivo, no sentido de que o programa aumentou a sensibilidade e a capacidade de respostas positivas nas suas interações com os seus filhos. 
Os estudos empíricos em diferentes contextos que buscam desenvolver, adaptar ou avaliar programas de treinamento parental ratificam a importância desse tema e a preocupação da comunidade científica com o desenvolvimento de intervenções dirigidas à parentalidade. A técnica de treinamento de pais é amplamente investigada buscando abranger as diversas variáveis de comportamento tanto dos filhos como dos pais e incentivar melhores formas de relacionamento entre os mesmos (Caleiro \& Silva, 2012). O treinamento de pais realizado em grupo tem se mostrado efetivo devido à possibilidade de troca de experiências entre os pais, o que contribui para maior aprendizado das orientações fornecidas ao longo da intervenção. A aplicação do treinamento em grupo também possibilita um alcance maior à população de forma mais econômica, tanto de recursos financeiros como humanos e, consequentemente, maior número de pessoas poderão beneficiar-se com a intervenção (Velasquez et al., 2010).

Considerando a importância do treinamento de pais e a necessidade de reunir informações que possibilitem a implementação dessa intervenção de forma eficaz, o objetivo deste estudo foi analisar as características metodológicas dos estudos empíricos sobre treinamento de pais, realizados em grupo. Pretendeu-se, ainda, descrever as técnicas empregadas nos treinamentos de pais descritos pelos estudos. Para isso, conduziu-se uma revisão sistemática da literatura nacional e internacional sobre o tema. Os estudos de revisão sistemática, organizando e discutindo investigações científicas, contribuem para o aprimoramento das práticas psicológicas, na medida em que descrevem métodos e técnicas relacionando-os com os resultados encontrados. Especificamente sobre o tema investigado, a expectativa é que os resultados possam servir de base para uma melhor adequação de programas aplicados ao contexto local, e para a escolha de técnicas adequadas ao objetivo da intervenção e à população alvo. Este estudo abrangeu artigos de 2006 a junho de 2014. Esse período foi definido considerando-se que o estudo de Bolsoni-Silva, Villas Boas, Romera e Silveira (2010) apresentou uma revisão sistemática com objeti- vo semelhante e que incluiu artigos nacionais e internacionais publicados até o ano de 2006.

\section{Método}

Foi realizada uma pesquisa baseada no método de revisão sistemática da literatura sobre o tema "treinamento parental", com os artigos publicados entre 2006 e junho de 2014. A consulta foi realizada entre julho e agosto de 2014, nas seguintes bases de dados: Literatura Latino-Americana e do Caribe em Ciências da Saúde (Lilacs), Scientific Electronic Library Online (SciELO), Medline (via Biblioteca Virtual em Saúde [BVS]) e PsycInfo. Os termos utilizados na busca nas bases Lilacs e Scielo foram: (a) treinamento ou intervenção e pais; (b) família ou pais e intervenção. Nas bases Medline e PsycInfo, foi usada a seguinte combinação: (a) parent training or family intervention. Tais palavras poderiam estar localizadas no título, resumo ou assunto. Também foram utilizados os seguintes limites na busca: ano de publicação; relato de casos, estudo de coorte, estudo de casos e controles; com seres humanos e adultos; inglês e espanhol; publicados em "peer reviewed journal only"; "empirical study"; "journal article". Ao todo, foram encontrados 423 artigos.

Os resumos dos 423 artigos foram lidos por dois pesquisadores independentes e foram aplicados os seguintes critérios de exclusão: (a) estudos teóricos ou de revisão sistemática da literatura; (b) estudos com crianças e adolescentes, sem, contudo, haver intervenção na família; (c) estudos repetidos. Restaram, então, 74 potenciais estudos para a análise dos dados. Destes, foram excluídos 47 artigos após a leitura na íntegra, por não satisfazerem os seguintes critérios de inclusão: (a) abordar treinamento ou orientação de pais; (b) apresentar um treinamento realizado em grupo. Por fim, o número final de artigos considerados para a fase de extração dos dados foi 27 , conforme indicado no fluxograma a seguir.

Dos estudos selecionados foram extraídos os seguintes dados: título do artigo, autores, ano de publicação, objetivo do estudo, público alvo, tamanho da amostra, delineamento, estratégias de intervenção e principais resultados. 
Consulta aos Bancos de Dados Lilacs e Scielo com os termos "treinamento ou intervenção e pais"; "família ou pais e intervenção"; e nas Bases Medline e PsycInfo com a combinação "parent training or family intervention".

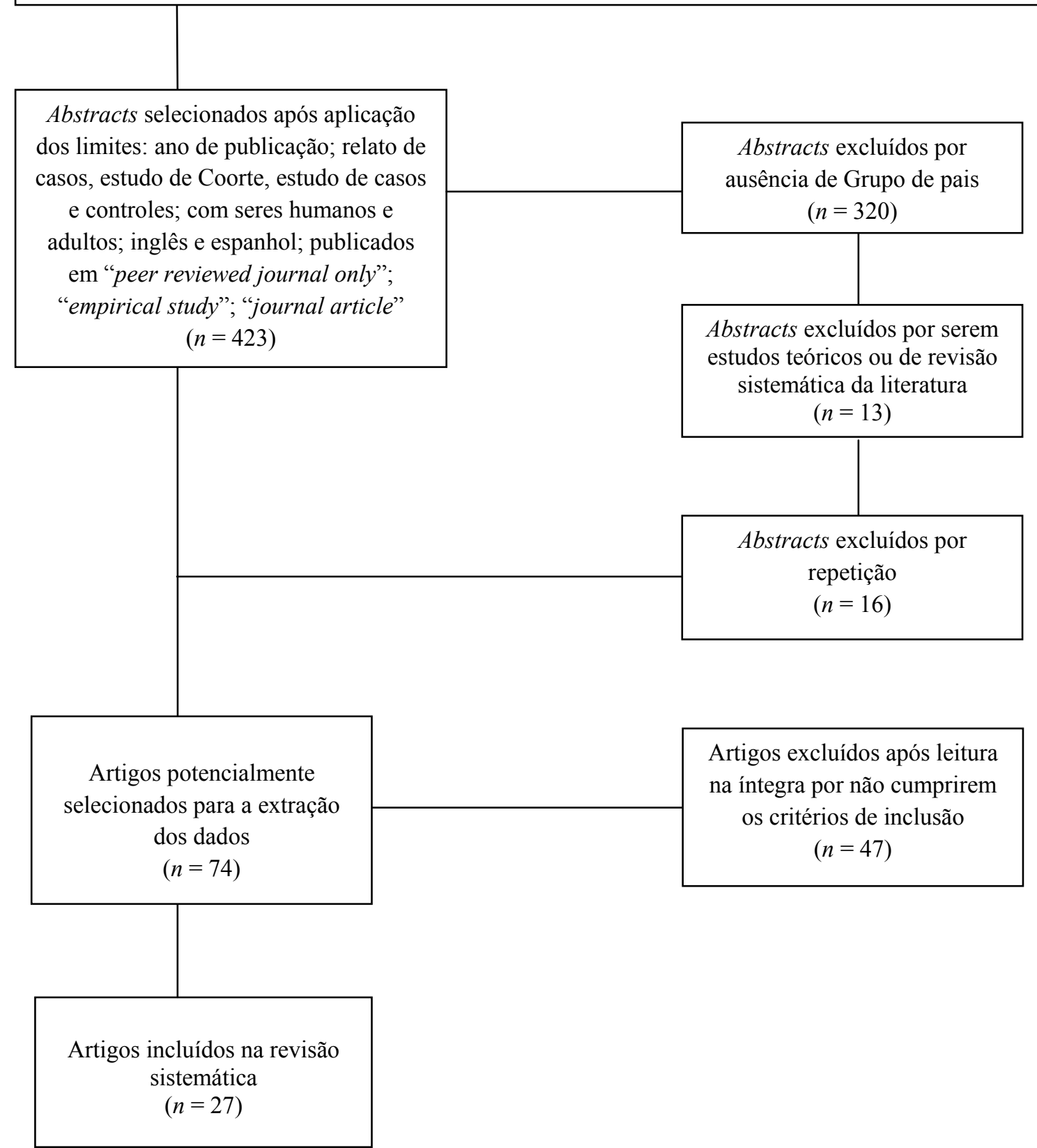

Figura 1. Fluxograma

\section{Resultados e Discussão}

A apresentação dos resultados será realizada de acordo com os objetivos do estudo, que foram analisar as características metodológicas dos estudos empíricos sobre treinamento de pais e apresentar as técnicas empregadas nos mesmos.

\section{Características Metodológicas}

A Tabela 1 apresenta as características metodológicas dos 27 estudos revisados, incluindo: nome dos autores e ano de publicação, delineamento, público alvo da intervenção, número de indivíduos que participaram da intervenção e do grupo controle (quando presente), e número total dos encontros referentes ao grupo que recebeu a intervenção. Ao longo do texto, a referência aos estudos se dará através da numeração da Tabela 1 . 
Tabela 1

Nome dos Autores e Ano de Publicação, Delineamento do Estudo, Público Alvo, Tamanho do Grupo de Intervenção e do Grupo Controle e Número de Encontros do Grupo de Intervenção

\begin{tabular}{|c|c|c|c|c|c|}
\hline Autores/ano & Delineamento & Público alvo & $N=$ intervenção & $N=$ grupo controle & $N=$ sessões \\
\hline $\begin{array}{l}\text { 1. Weber, Brandenburg, } \\
\text { \& Salvador (2006) }\end{array}$ & $\begin{array}{c}\text { Quase } \\
\text { experimental }\end{array}$ & Pais & & pais & 08 \\
\hline $\begin{array}{l}\text { 2. Rodrigo, Correa, Máiquez, } \\
\text { Martín, \& Rodríguez (2006) }\end{array}$ & $\mathrm{NC}$ & Pais & 144 Pais & 155 Pais & 21 \\
\hline 3. Grskovic \& Goetze (2008) & $\begin{array}{l}\text { Ensaio clínico } \\
\text { randomizado }\end{array}$ & $\begin{array}{l}\text { Mães / } \\
\text { Crianças }\end{array}$ & 15 mães & 18 mães & 08 \\
\hline $\begin{array}{l}\text { 4. Nix, Bierman, } \\
\text { \& McMahon (2009) }\end{array}$ & $\begin{array}{l}\text { Ensaio clínico } \\
\text { randomizado }\end{array}$ & Pais & 445 pais & $\mathrm{NC}$ & 22 \\
\hline $\begin{array}{l}\text { 5. Haywood \& McCann } \\
\text { (2009) }\end{array}$ & $\mathrm{NC}$ & Famílias & \multicolumn{2}{|c|}{12 famílias } & 04 \\
\hline $\begin{array}{l}\text { 6. Matsumoto, Sofronoff, } \\
\text { \& Sanders (2010) }\end{array}$ & $\begin{array}{l}\text { Ensaio clínico } \\
\text { randomizado }\end{array}$ & Famílias & 28 Famílias & 26 famílias & $\mathrm{NC}$ \\
\hline 7. Edwards et al. (2010) & $\begin{array}{l}\text { Estudo } \\
\text { etnográfico }\end{array}$ & Pais & \multicolumn{2}{|c|}{5 pais } & 10 \\
\hline $\begin{array}{l}\text { 8. Berge, Law, Johnson, } \\
\text { \& Wells (2010) }\end{array}$ & $\mathrm{NC}$ & $\begin{array}{l}\text { Pais / } \\
\text { crianças }\end{array}$ & \multicolumn{2}{|c|}{35 pais } & 12 \\
\hline $\begin{array}{l}\text { 9. Cowan, Cowan, \& Barry } \\
\text { (2011) }\end{array}$ & $\begin{array}{l}\text { Ensaio clínico } \\
\text { randomizado }\end{array}$ & Casais & 74 casais & 26 casais & $16 \mathrm{~s}$ \\
\hline $\begin{array}{l}\text { 10. Sanders, Stallman, } \\
\text { \& McHale (2011) }\end{array}$ & $\begin{array}{l}\text { Ensaio clínico } \\
\text { randomizado }\end{array}$ & Pais & 62 pais & 59 pais & 04 \\
\hline 11. Coser et al. (2011) & $\begin{array}{l}\text { Quase } \\
\text { experimental }\end{array}$ & Pais & \multicolumn{2}{|c|}{3 pais } & 08 \\
\hline $\begin{array}{l}\text { 12. Herman, Borden, Reinke, } \\
\text { \& Webster-Stratton (2011) }\end{array}$ & $\begin{array}{l}\text { Ensaio clínico } \\
\text { randomizado }\end{array}$ & $\begin{array}{c}\text { Pais / } \\
\text { Crianças }\end{array}$ & 31 pais & 26 pais & 24 \\
\hline 13. Boutelle et al. (2011) & $\begin{array}{l}\text { Ensaio clínico } \\
\text { randomizado }\end{array}$ & $\begin{array}{c}\text { Pais / } \\
\text { Crianças }\end{array}$ & 18 díades & 18 díades & 08 \\
\hline 14. McGilloway et al. (2012) & $\begin{array}{l}\text { Ensaio clínico } \\
\text { randomizado }\end{array}$ & Pais & 103 pais & 46 pais & 14 \\
\hline 15. Power et al. (2012) & $\begin{array}{l}\text { Ensaio clínico } \\
\text { randomizado }\end{array}$ & $\begin{array}{c}\text { Pais / } \\
\text { Crianças }\end{array}$ & 100 famílias & 99 famílias & 12 \\
\hline $\begin{array}{l}\text { 16. Posthumus, Raaijmakers, } \\
\text { Maassen, van Engeland, } \\
\text { \& Matthys (2012) }\end{array}$ & Caso controle & Pais & 72 pais & 72 pais & 18 \\
\hline 17. Forehand et al. (2012) & $\begin{array}{l}\text { Ensaio clínico } \\
\text { randomizado }\end{array}$ & Famílias & 90 famílias & 90 famílias & 12 \\
\hline 18. Pfiffner et al. (2013) & $\begin{array}{l}\text { Ensaio clínico } \\
\text { randomizado }\end{array}$ & $\begin{array}{c}\text { Pais / } \\
\text { Crianças }\end{array}$ & \multicolumn{2}{|c|}{57 Crianças } & 10 \\
\hline 19. Lochrie et al. (2013) & $\begin{array}{l}\text { Ensaio clínico } \\
\text { randomizado }\end{array}$ & Famílias & 65 famílias & 65 famílias & 14 \\
\hline $\begin{array}{l}\text { 20. Chronis-Tuscano et al. } \\
\text { (2013) }\end{array}$ & $\begin{array}{l}\text { Ensaio clínico } \\
\text { randomizado }\end{array}$ & Mães & 51 mães & 47 mães & 14 \\
\hline $\begin{array}{l}\text { 21. Fabrizio, Stewart, Ip, } \\
\text { \& Lam (2014) }\end{array}$ & $\begin{array}{l}\text { Ensaio clínico } \\
\text { randomizado }\end{array}$ & Pais & 131 pais & 118 pais & 04 \\
\hline $\begin{array}{l}\text { 22. Gewirtz, Pinna, Hanson, } \\
\text { \& Brockberg (2014) }\end{array}$ & $\begin{array}{l}\text { Ensaio clínico } \\
\text { randomizado }\end{array}$ & Famílias & 42 famílias & 27 famílias & 14 \\
\hline
\end{tabular}


23. Burke et al. (2014)

24. Wong et al. (2014)

25. Armistead et al. (2014)

26. Santiago, Lennon, Fuller, Brewer, \& Kataoka (2014)

27. van der Ende, van Busschbach, Nicholson, Korevaar, \& van Weeghel (2014)
$\mathrm{NC}$

Ensaio clínico randomizado

Ensaio clínico randomizado

Quase experimental

Ensaio clínico randomizado
Pais

Pais

Pais

Pais

Pais
11 pais

05

09

06

12

32 díades $\quad 32$ díades

15 pais

Notas. $N=$ Número total $/ \mathrm{NC}=$ Não consta no artigo.

A Figura 2 mostra o delineamento dos estudos de acordo com os anos de publicação. Observa-se que o número de artigos publicados sobre treinamento de pais vem aumentando ao longo dos anos, de forma geral. Com exceção dos anos de 2007 e 2013, que mostram uma pe- quena queda quanto ao número de publicações, a quantidade de estudos publicados amplia-se anualmente, com um maior número de estudos publicados em 2014 (sete estudos). Considerando o período da análise, a média de publicações foi de três artigos por ano $(M=3)$.

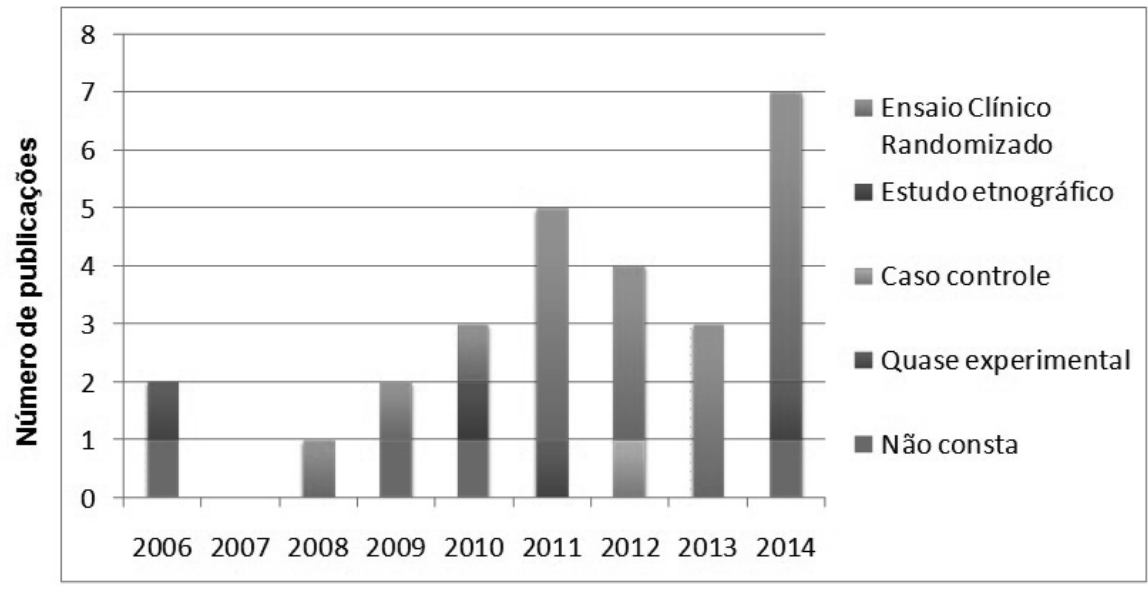

Ano de publicação

Figura 2. Delineamento de acordo com o ano de publicação dos estudos.

Com relação ao delineamento, nota-se maior frequência de ensaios clínicos randomizados (ECR). O achado vai ao encontro dos dados levantados por Bolsoni-Silva et al. (2010), que perceberam o aumento da utilização de ECR a partir do ano 2001. Do total de artigos selecionados, 18 utilizaram esse delineamento $(3,4,6,9$, $10,12,13,14,15,17,18,19,20,21,22,24,25$, 27), representando $66,7 \%$ da amostra. Por se tratar de estudos que almejam avaliar os efeitos de programas de intervenção, esse tipo de delineamento se faz vantajoso, uma vez que possibilita o controle de um maior número de vieses, aumentando a validade interna dos estudos (Campbell \& Stanley, 1979). Além do ECR, foram encontrados três estudos desenvolvidos através do desenho quase experimental $(1,11,26)$, um caso controle (16) e um estudo etnográfico (7).

Sobre o público alvo, que consta na Tabela 1, o termo Família refere-se à tríade pai-mãe-filho, que realizaram a intervenção juntos, em grupo; Pais indica que pais e mães foram convidados a participar da intervenção; o termo Pais e crianças trata das sessões que ocorreram 
tanto com os pais, quanto com os filhos, porém em grupos separados. Nos três casos, ambos os genitores foram convidados a participar do estudo, mas poderia comparecer apenas um representante do casal, ficando a critério da família a disponibilidade para tal.

Concluímos que o maior número de estudos teve como público alvo somente os pais, representando $52 \%$ da amostra $(1,2,4,7,10,11,12$, $16,21,23,24,25,26,27)$, seguido de estudos que intervém com os pais e os filhos, porém em grupos separados, com $22 \%(3,8,12,13,15$, 18). Já as pesquisas que envolvem a família têm menor frequência, com $19 \%(5,6,17,19,22)$. Um estudo encontrado teve como público alvo especificamente as mães com depressão ou com histórico da doença (20), enquanto outro se referia a casais (9), completando o número total dos estudos encontrados (7\%).

Ainda sobre o público alvo, é relevante citar que três artigos incluíram os professores em algum momento da intervenção, nos casos de transtornos disruptivos: ou em reuniões individuais - de 2 a 3 encontros - com os pais e a criança $(15,18)$, ou participando de um treinamento em grupo para minimizar os sintomas internalizantes dos jovens, visando também construir um processo de socialização consistente entre a casa e a escola (12). Ademais, três estudos tiveram um componente individual para pais e filhos (24, $25,26)$, onde foram realizados alguns encontros somente com a díade, na intenção de complementar as sessões grupais. Ao mesmo tempo, chama a atenção o fato de que apesar de pais e mães serem convidados a participar dos programas, a predominância da participação nos grupos é feminina, chegando a atingir mais de $90 \%$ da $\operatorname{amostra}(2,14)$.

No que se refere ao tamanho da amostra utilizada nas pesquisas, houve variação entre três e 513 sujeitos no grupo de intervenção, e entre 15 e 269 no grupo controle. Foram encontradas diferenças em termos de nomenclatura, pois alguns artigos referem-se ao número total de famílias participantes, enquanto outros apresentam o número total de sujeitos da amostra. Por isso, na Tabela 1, optamos por manter a classificação dos autores.
A presença de um grupo controle de lista de espera foi evidenciada em 12 pesquisas, representando $44 \%$ da amostra $(2,3,4,6,10,14$, $16,19,22,24,25,27)$; outros três estudos (11\%) incluíam dois ou mais grupos de intervenção, além do grupo controle $(9,12,21)$; cinco artigos (19\%) não têm grupo controle, mas apresentam dois ou mais grupos que sofreram alguma intervenção para comparação dos resultados $(13,15$, $17,20,26)$; e sete deles (26\%) não utilizaram a condição controle $(1,5,7,8,11,18,23)$. Segundo Cozby (2003), o uso de delineamentos experimentais envolvendo grupos controle aumenta a confiabilidade dos resultados observados. Os estudos que não utilizaram grupo controle $(1,8$, $11,18,23)$ discutiram essa característica como uma limitação, pois apesar de se verificar modificações nos participantes que experienciaram a intervenção, existem dificuldades metodológicas para avaliar o papel dessa nos resultados encontrados.

Observou-se a ocorrência do follow-up para avaliar longitudinalmente os resultados dos programas investigados, apresentando variação de três meses a 10 anos. O mais utilizado é o follow-up de até seis meses após a intervenção (22\% dos estudos). Do total, 18 estudos $(66,7 \%)$ utilizaram a avaliação longitudinal $(5,9,10,12$, $13,14,15,16,17,19,20,21,22,23,24,25,26$, $27)$, enquanto nove $(33,3 \%)$ realizaram somente a avaliação logo após o término dos grupos $(1,2$, $3,4,6,7,8,11,18)$, entre eles os dois estudos nacionais analisados. A ausência de follow-up impede a verificação da manutenção das mudanças ocorridas nos participantes após a intervenção. Esse dado é importante para que se possa avaliar a eficácia dos grupos de orientação a pais. $\mathrm{O}$ fato de estarem incluídos, entre os estudos que não utilizaram follow-up, os dois trabalhos nacionais remete a necessidade de qualificação das investigações nacionais quanto a esse aspecto.

Os resultados encontrados acerca das características metodológicas dos estudos que foram incluídos nessa revisão sugerem uma preocupação com o rigor no método científico. A maior frequência de estudos que utilizaram ECR, grupo controle e realizaram follow-up evidenciam tal preocupação. 


\section{Características da Intervenção}

Países. A diversidade dos países nos quais os estudos ocorreram indica que as intervenções grupais, especialmente sobre práticas educativas e transtornos disruptivos, são uma grande preocupação atual. Destaca-se que, dos 27 estudos encontrados na revisão sistemática sobre treinamento de pais que atenderam aos critérios de inclusão e de exclusão, apenas dois deles são brasileiros $(1,11)$, identificando uma lacuna na literatura nacional sobre o tema. Em contrapartida, o país que mais se destacou em termos de número de publicação foram os Estados Unidos, com 13 publicações $(4,7,8,9,12,13,15,17$, $18,19,20,22,26)$. Também foram encontrados estudos na Espanha (2); Alemanha (3); Japão (6); Austrália (10, 23); China (21); México (24); África do Sul (25); Holanda (16, 27); Irlanda (14); e Reino Unido (5).

Número de Encontros. O número de encontros do grupo variou de quatro a 24 , e não foi encontrada relação com o público alvo, pois não se manteve um padrão em nenhuma das categorias utilizadas. Quanto à periodicidade das sessões, em 20 dos estudos os encontros realizaram-se semanalmente $(1,2,4,5,7,8,9,12,13,14,15$, $16,18,20,21,22,23,24,25,27)$; dois deles apresentaram as sessões iniciais semanalmente, seguidas por sessões quinzenais e mensais (17, 19); um deles foi quinzenal (10); em um os encontros se deram duas vezes por semana (11); um estudo teve a periodicidade de quatro vezes por semana, no qual os oito encontros foram realizados em duas semanas (3); por fim, a periodicidade das sessões não constava em duas das pesquisas $(6,26)$.

\section{Objetivo da Intervenção}

As pesquisas que abordam treinamentos específicos com o objetivo de aprimorar as práticas educativas parentais, visando resultados positivos na relação entre pais e filhos, foram as mais frequentes, como mostra a Figura 3, com sete artigos analisados $(1,6,7,10,11,21,22)$. Os estudos relativos a Transtornos Disruptivos foram igualmente significantes, demonstrando preocupação a nível científico pelos transtornos de comportamento $(4,8,14,16,18)$ e Transtorno do Déficit de Atenção com Hiperatividade (TDAH; 15, 18). Também foram encontrados cinco estudos de orientação para pais portadores de psicopatologia, tais como depressão e ansiedade $(3,20,23,24,27)$. Quatro estudos apresentaram enfoque preventivo $(2,9,17,25)$, sendo um deles com orientações a um grupo de mães que habitam em uma comunidade de alto risco psicossocial relacionadas a cuidados com a saúde e desenvolvimento de seus filhos; outro estudo realizado com casais com o objetivo de aboradar questões relacionadas à relação dos mesmos, a fim de incentivar a harmonia familiar e evitar que os problemas do casal afetem a relação com os filhos; outro estudo realizado

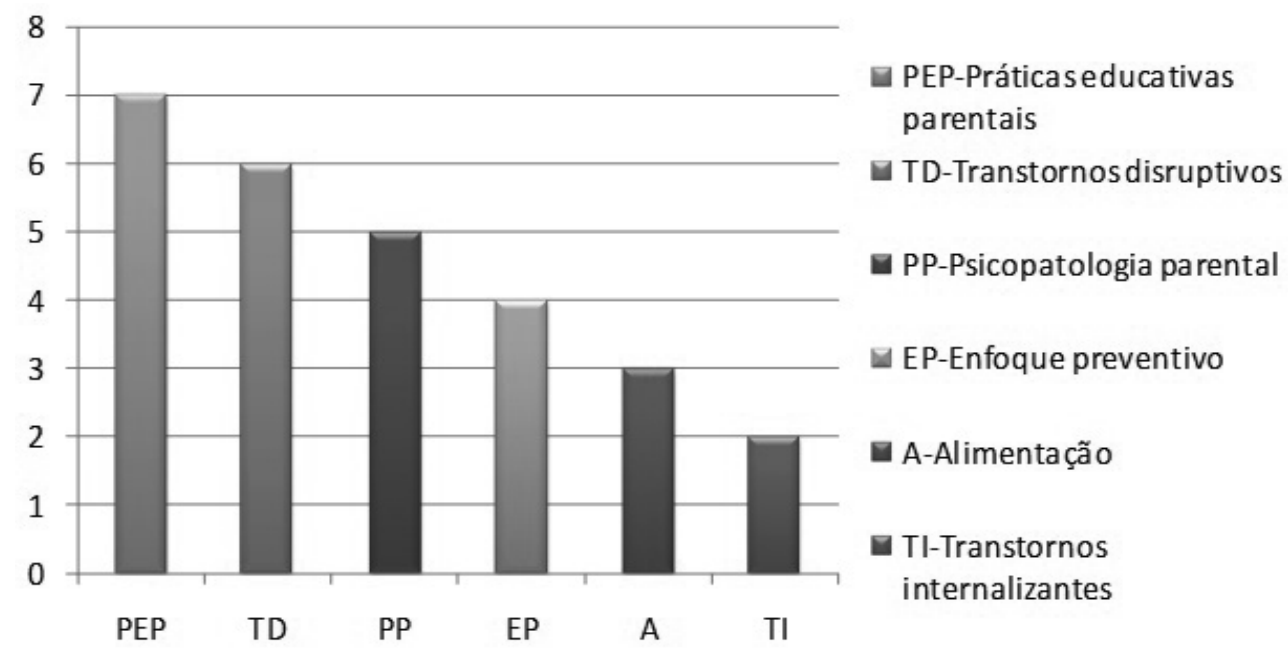

Figura 3. Objetivo da intervenção 
para avaliação do ensino de técnicas parentais a um grupo composto por pais que apresentavam sintomas depressivos com objetivo de prevenir que os filhos desenvolvessem problemas comportamentais; e também um estudo realizado com um grupo para orientação de pais de adolescentes em relação à prevenção do contágio por HIV, a fim de aprimorar o diálogo entre pais e filhos sobre assuntos relacionados à sexualidade e prevenção de HIV. Por fim, três estudos eram referentes a problemas alimentares/obesidade $(5$, $13,19)$ e dois tratavam de transtornos internalizantes $(12,26)$.

\section{Técnicas Identificadas}

No que diz respeito às técnicas utilizadas nos treinamentos de pais avaliados, verificou-se que a maioria está relacionada à terapia cognitivo-comportamental, conforme ilustrado na Figura 4. Dentre os artigos que se propuseram a explicá-las de forma clara, notou-se o uso de materiais audiovisuais para modelagem e feedback, visando enriquecer os debates e proporcionar o envolvimento prático dos pais quanto ao conteúdo e técnicas aprendidas. Também foram utilizados o treino de resolução de problemas, a tarefa de casa, e a discussão dos temas abordados em pequenos grupos.

Analisando as técnicas de acordo com o objetivo dos estudos (Figura 5), verificou-se que, de forma geral, não foi encontrado nenhum tipo de padronização dos programas, quanto a número de encontros, número de sessões ou uso de follow-up. Entretanto, algumas técnicas se repetem em estudos com o mesmo objetivo: (a) Práticas educativas parentais: feedback $(7,11)$, consistindo na correção de atitudes inadequadas por parte dos pais participantes dos grupos em relação à educação de seus filhos, assim como no reforçamento de postura adequada; role play $(11,22)$, onde os pais foram incentivados a assumirem e reproduzirem o papel de seus próprios filhos diante do grupo de participantes a fim de reviver situações em que o comportamento inadequado dos filhos se manifestou, e assim, possibilitar maior compreensão a respeito do comportamento de seus filhos e aprimorar o vínculo afetivo e a relação entre pais e filhos; e discussões $(1,22)$ nas quais foram debatidos entre os participantes suas dúvidas, experiências e formas corretas de lidar com o comportamento de seus filhos; (b) Psicopatologia parental: feedback $(23,3)$; (c) Problemas de alimentação: resolução de problemas $(19,13)$, que consistiu no ensino aos participantes de dieta adequada e diversos hábitos saudáveis, a fim de promover o emagrecimento e saúde de pais e filhos; (d) Transtornos disruptivos: role play $(16,14,4)$ e modelagem $(14,4)$ onde foram ensinadas práticas educativas positivas ligadas ao comportamento dos pais as quais poderiam ser transferidas ao comportamento de seus filhos.

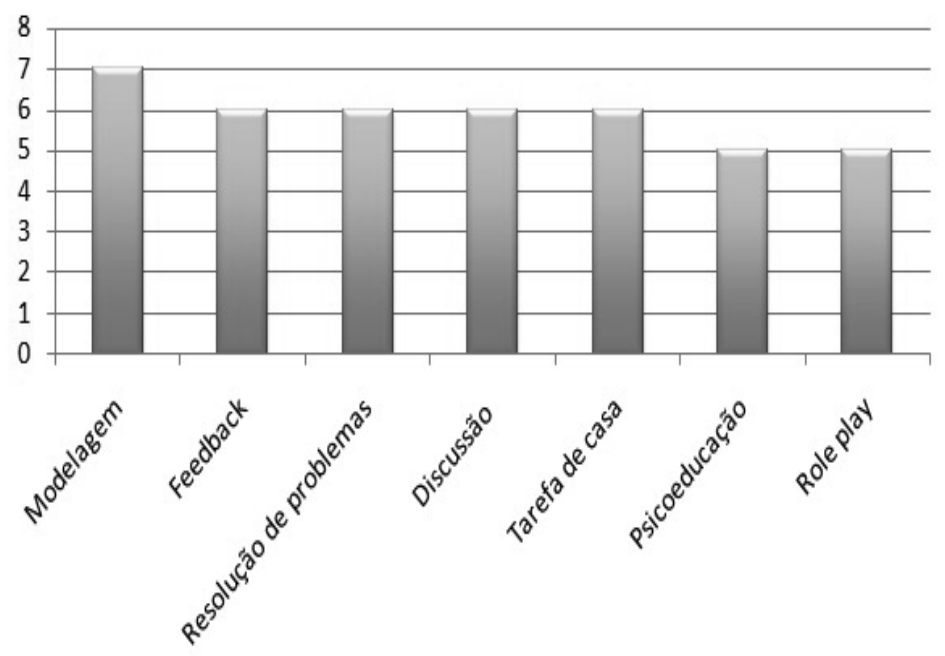

Figura 4. Técnicas mais utilizadas. 


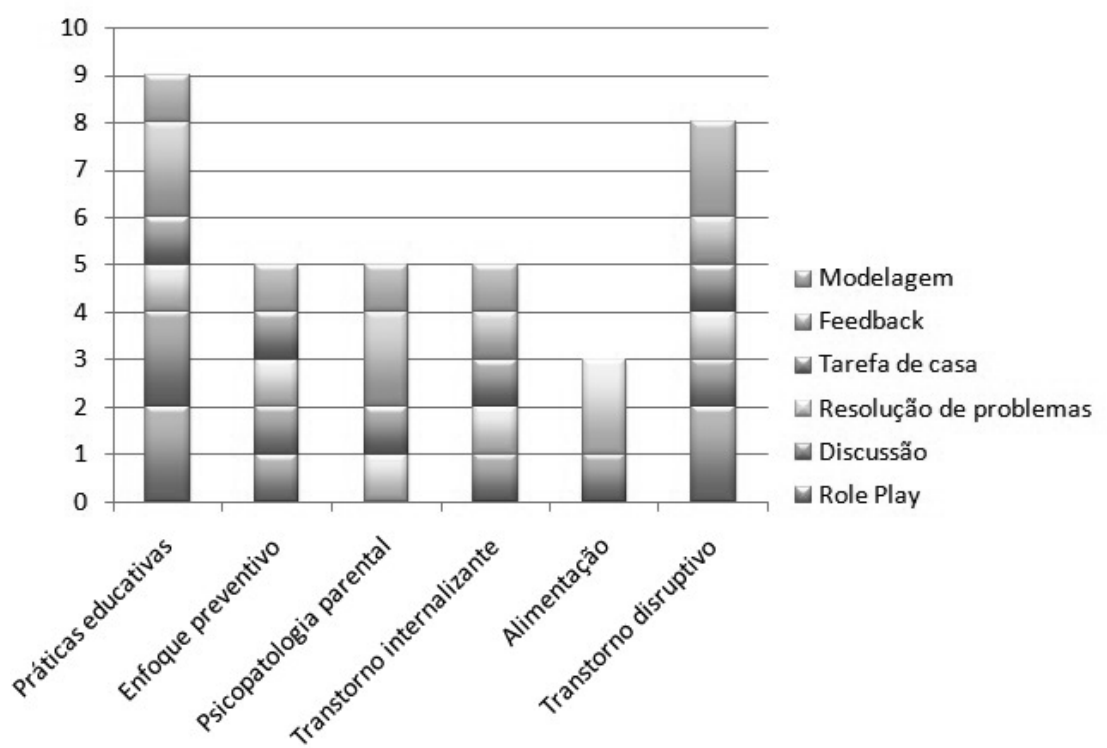

Figura 5. Técnicas mais utilizadas de acordo com o objetivo dos estudos.

O uso de chamadas telefônicas individuais aos participantes para complementar as sessões de grupo ocorreu em alguns estudos, servindo como suporte do coordenador e para refinar as intervenções, quando necessário $(10,14,15)$. Não obstante, as chamadas telefônicas também ocorreram no caso de ausência dos participantes em algum dos encontros. Os coordenadores dos grupos realizaram telefonemas (de 20 a 30 minutos) visando informar os sujeitos sobre os temas trabalhados, para que não houvesse prejuízo quanto ao conteúdo e ao seu seguimento no grupo (21). Outra estratégia interessante foi encontrada no estudo de Gewirtz et al. (2014), que desenvolveu um conjunto de ferramentas online para o programa, que poderia ser acessado a partir de casa a qualquer momento servindo como reforço das sessões. A base online incluía vídeos sobre as competências parentais positivas e exercícios de conscientização ensinados, ficando disponíveis para download aos participantes, como incentivo à prática diária.

Observa-se, em 33\% da amostra deste estudo, que houve algum tipo de compensação financeira às famílias para incentivar a participação $(4,6,8,14,15,18,19,21,22)$. As famílias foram remuneradas em dinheiro a cada etapa de coleta dos dados, ou foi, no mínimo, fornecido reembolso dos gastos referentes à participação na pesquisa, como transporte, alimentação e creche para as crianças que não frequentam os grupos. Essa prática não é permitida no Brasil, mas pode-se inferir pelo maior número de publicações internacionais que este pode ser um fator que contribui para permanência dos sujeitos ao longo da pesquisa.

Principais Resultados. Sobre os principais resultados dos artigos analisados, notou-se que o maior número de estudos alcançou mudanças nas práticas parentais $(1,2,4,6,10,11,17,25$, 26,27 ), representando $37 \%$ da amostra, seguido por resultados na redução de problemas de comportamento das crianças e melhora nas práticas parentais $(3,15,16,20,24)$, com $18,5 \%$. Com uma porcentagem similar $(15 \%)$, foram encontradas pesquisas que tratam de melhoras no comportamento, juntamente com mudanças nas relações familiares $(7,8,9,14)$. Em 7\% da amostra, os resultados obtidos foram em relação à redução de problemas de comportamento (12, 18); e por fim, um estudo alcançou resultados na melhora das práticas e na relação familiar (21), o equivalente a $4 \%$. Trabalhos que alcançaram resultados em categorias não contempladas no gráfico foram inseridos na categoria outros (5, 13, 19, 22, 23), alcançando, também, 18,5\% do total da amostra.

Portanto, a análise dos principais resultados encontrados aponta que a totalidade dos estudos realizados alcançou resultados positivos, en- 
quanto a maioria focalizou as competências dos pais, o que tende a contribuir para a manutenção dos resultados alcançados com as crianças.

\section{Considerações Finais}

O objetivo deste artigo foi realizar a análise das características metodológicas dos estudos empíricos sobre treinamento de pais e apresentar as técnicas empregadas. Verificou-se um número significativo de estudos sobre treinamento parental na literatura internacional. Entretanto, poucos descrevem a intervenção, em termos de metodologia, técnicas e recursos, com clareza. Se por um lado, os delineamentos escolhidos pela maioria dos estudos (ECR, grupo controle e follow-up) contribuem para a confiança nos resultados quanto à eficácia da intervenção, por outro lado, a ausência de detalhamento acerca da intervenção pesquisada dificulta a replicação dos estudos e limita a identificação das técnicas que produzem melhores resultados.

Técnicas padronizadas como utilização de vídeo, role play, modelagem e resolução de problemas foram as mais utilizadas e contribuem para efeitos satisfatórios, como visto nos resultados encontrados. Ressaltam-se a utilização de chamadas telefônicas e o uso da internet como meios alternativos de acesso aos participantes. Mesmo que estas sejam maneiras difíceis de serem generalizadas a todas as populações, destacam-se como meios interessantes para manter os participantes na intervenção e poderiam ser mais explorados em estudos futuros.

Os resultados desta pesquisa sugerem um interesse crescente e global pela área de treinamento parental, como se pôde observar por meio do número de publicação ao longo dos anos e da diversidade de países que apresentam estudos neste contexto. Contudo, observamos que a quantidade de pesquisas preventivas ainda é pequena, quando comparada aos estudos que enfocam transtornos ou problemas já presentes a nível individual e/ou familiar. A preocupação em promover intervenções preventivas também deve ser contínua, principalmente no âmbito da saúde pública, em função de suas vantagens humanas e econômicas (Velasquez et al., 2010).
Ainda, os programas de orientação parental desenvolvidos e apresentados neste estudo, de acordo com os resultados obtidos, alcançaram efeitos positivos, sugerindo principalmente implicações em relação a mudanças positivas no repertório de habilidades dos pais.

Quanto aos estudos nacionais, identificouse um pequeno número de pesquisas que cumpriram os critérios de inclusão. Dois estudos nacionais foram incluídos nessa análise e nenhum realizou uma avaliação dos resultados a longo prazo (follow-up). Esse dado indica a necessidade de mais investigação sobre $o$ tema no Brasil. Possivelmente, isso represente a superação de dificuldades metodológicas como desenvolver estudos com um tamanho amostral representativo, manter os participantes ao longo da intervenção e acompanha-los após o término dessa. Nesse sentido, entende-se que os estudos de revisão sistemática da literatura podem contribuir para que pesquisadores possam identificar estratégias metodológicas e intervenções terapêuticas que viabilizam tanto a construção de projetos de pesquisa, quanto à atuação psicológica.

\section{Referências}

Armistead, L., Cook, S., Skinner, D., Toefy, Y., Anthony, E. R., Zimmerman, L., ...Chow, L. (2014). Preliminary results from a family-based HIV prevention intervention for South African youth. Health Psychology, 33(7), 668-676. doi: $10.1037 /$ hea0000067

Bagner, D. M. (2013). Father's role in parent training for children with developmental delay. Journal of Family Psychology, 27(4), 650-657. doi:10.1037/a0033465

Berge, J. M., Law, D. D., Johnson, J., \& Wells, M. G. (2010). Effectiveness of a psychoeducational parenting group on child, parent, and family behavior: A pilot study in a family practice clinic with an underserved population. Families, Systems, \& Health, 28(3), 224-235. doi:10.1037/ a0020907

Bolsoni-Silva, A. T., \& Marturano, E. M. (2002). Práticas educativas e problemas de comportamento: Uma análise à luz das habilidades sociais. Estudos de Psicologia (Natal), 7, 227-235. 
Bolsoni-Silva, A. T., Silveira, F. F., \& Marturano, E. M. (2008). Promovendo habilidades sociais educativas parentais na prevenção de problemas de comportamento. Revista Brasileira de Terapia Comportamental Cognitiva, 10(2), 125-142.

Bolsoni-Silva, A. T., Silveira, F. F., \& Ribeiro, D. C. (2008). Avaliação dos efeitos de uma intervenção com mães/cuidadoras: Contribuições do treinamento em habilidades sociais. Contextos Clínicos, 1(1), 19-27.

Bolsoni-Silva, A. T., Villas Boas, A. C. V. B., Romera, V. B., \& Silveira, F. F. (2010). Caracterização de programas de intervenção com crianças e/ou adolescentes. Arquivos Brasileiros de Psicologia, 62(1), 104-118.

Boutelle, K. N., Zucker, N. L., Peterson, C. B., Rydell, S. A., Cafri, G., \& Harnack, L. (2011). Two novel treatments to reduce overeating in overweight children: A randomized controlled trial. Journal of Consulting and Clinical Psychology, 79(6), 759-771. doi:10.1037/a0025713

Brennan, L. M., Shelleby, E. C., Shaw, D. S., Gardner, F., Dishion, T. J., \& Wilson, M. (2013). Indirect effects of the family check-up on school-age academic achievement through improvements in parenting in early childhood. Journal of Educational Psychology, 105(3), 762-773. doi:10.1037/a0032096

Burke, K., Muscara, F., McCarthy, M., Dimovski, A., Hearps, S., Anderson, V., \& Walser, R. (2014). Adapting acceptance and commitment therapy for parents of children with life-threatening illness: Pilot study. Families, Systems, \& Health, 32(1), 122-127. doi:10.1037/fsh0000012

Caleiro, F. M., \& Silva, R. S. (2012). Técnicas de modificação do comportamento de crianças com treinamento de pais. Encontro Revista de Psicologia, 15(23), 129-142.

Campbell, D. T., \& Stanley, J. C. (1979). Delineamentos experimentais e quase-experimentais de pesquisa. São Paulo, SP: Editora da Universidade de São Paulo.

Chronis-Tuscano, A., Clarke, T. L., O’Brien, K. A., Raggi, V. L., Diaz, Y., Mintz, A. D., ... Lewinsohn, P. (2013). Development and preliminary evaluation of an integrated treatment targeting parenting and depressive symptoms in mothers of children with attention-deficit/ hyperactivity disorder. Journal of Consulting and Clinical Psychology, 81(5), 918-925. doi:10.1037/a0032112
Coser, D. S., Cortegoso, A. L., \& Gil, M. S. C. A. (2011). Promoção de comportamentos de estudo em crianças: Resultados de um programa de ensino para pais e responsáveis. Revista Brasileira de Terapia Comportamental e Cognitiva, 13(2), 58-78.

Cowan, C. P., Cowan, P. A., \& Barry, J. (2011). Couples' groups for parents of preschoolers: Ten-year outcomes of a randomized trial. Journal of Family Psychology, 25(2), 240-250. doi:10.1037/a0023003

Cozby, P. C. (2003). Métodos de pesquisa em ciências do comportamento. São Paulo, SP: Atlas.

Donohue, B., Azrin, N. H., Bradshaw, K., Van Hasselt, V. B., Cross, C. L., Urgelles, J., ... Allen, D. N. (2014). A controlled evaluation of family behavior therapy in concurrent child neglect and drug abuse. Journal of Consulting and Clinical Psychology, 82(4), 706-720. doi:10.1037/a0036920

Edwards, N. A., Sullivan, J. M., Meany-Walen, K., \& Kantor, K. R. (2010). Child parent relationship training: Parents' perceptions of process and outcome. International Journal of Play Therapy, 19(3), 159-173. doi:10.1037/a0019409

Fabrizio, C. S., Stewart, S. M., Ip, A. K. Y., \& Lam, T. H. (2014). Enhancing the parentchild relationship: A Hong Kong communitybased randomized controlled trial. Journal of Family Psychology, 28(1), 42-53. doi:10.1037/ a0035275

Forehand, R., Thigpen, J. C., Parent, J., Hardcastle, E. J., Bettis, A., \& Compas, B. E. (2012). The role of parent depressive symptoms in positive and negative parenting in a preventive intervention. Journal of Family Psychology, 26(4), 532541. doi:10.1037/a0028406

Gewirtz, A. H., Pinna, K. L. M., Hanson, S. K., \& Brockberg, D. (2014). Promoting parenting to support reintegrating military families: After deployment, adaptive parenting tools. Psychological Services, 11(1), 31-40. doi:10.1037/ a0034134

Grskovic, J. A., \& Goetze, H. (2008). Short-term filial therapy with German mothers: Findings from a controlled study. International Journal of Play Therapy, 17(1), 39-51. doi:10.1037/15556824.17.1.39

Hauth-Charlier, S., \& Clément, C. (2014). Abord dimensionnel de l'efficacité d'un programme 
d'entraînement aux habiletés parentales dans le contexte du TDA/H. Revue Canadienne des Sciences du Comportement, 46(2), 107-116. doi:10.1037/a0034470

Haywood, P., \& McCann, J. (2009). A brief group intervention for young children with feeding problems. Clinical Child Psychology and Psychiatry, 14(3), 361-72.

Herman, K. C., Borden, L. A., Reinke, W. M., \& Webster-Stratton, C. (2011). The impact of the Incredible Years parent, child, and teacher training programs on children's co-occurring internalizing symptoms. School Psychology Quarterly, 26(3), 189-201. doi:10.1037/a0025228

Hodgetts, S., Savage, A., \& McConnell, D. (2013). Experience and outcomes of stepping stones triple $\mathrm{P}$ for families of children with autism. Research in developmental Disabilies, 34(9), 2572 2585 .

Jouriles, E. N., McDonald, R., Rosenfield, D., Norwood, W. D., Spiller, L., Stephens, N., ... Ehrensaft, M. (2010). Improving parenting in families referred for child maltreatment: A randomized controlled trial examining effects of Project Support. Journal of Family Psychology, 24(3), 328-338. doi:10.1037/a0019281

Kazdin, A. E. (1997). Parent management training: Evidence, outcomes and issues. Journal of the American Academy of Child \& Adolescent Psychiatry, 36(10), 1349-1356. doi:10.1097/00004583-199710000-00016

Kazdin, A. E. (2003). Psychotherapy for children and adolescents. Annual Review of Psychology, 54, 253-276

Lee, L., Griffiths, C., Glossop, P., \& Eapen, V. (2010). The Boomerangs Parenting Program for Aboriginal parents and their young children. Australas Psychiatry, 18(6), 527-533.

Lochrie, A. S., Wysocki, T., Hossain, J., Milkes, A., Antal, H; Buckloh, L., ...Lang, J. (2013). The effects of a family-based intervention (FBI) for overweight/obese children on health and psychological functioning. Clinical Practice in Pediatric Psychology, 1(2), 159-170. doi:10.1037/ cpp0000020

Loeber, R., \& Hay, D. (1997). Key issues in the development of aggression and violence from childhood to early adulthood. Annual Review of Psychology, 48, 371-410.
Marinho, M. L., \& Silvares, E. F. (2001). Modelos de orientação a pais de crianças com queixas diversificadas. In R. C. Wielenska (Ed.), Sobre comportamento e cognição - Questionando e ampliando a teoria e as intervenções clínicas e em outros contextos (pp. 165-178). Santo André, SP: Arbytes.

Matsumoto, Y., Sofronoff, K., \& Sanders, M. R. (2010). Investigation of the effectiveness and social validity of the Triple P Positive Parenting Program in Japanese society. Journal of Family Psychology, 24(1), 87-91. doi:10.1037/ a0018181

McGilloway, S., Ni Mhaille, G., Bywater, T., Furlong, M., Leckey, Y., Kelly, P., ...Donnelly, M. (2012). A parenting intervention for childhood behavioral problems: A randomized controlled trial in disadvantaged community-based settings. Journal of Consulting \& Clinical Psychology, 80(1), 116-127.

McMahon, R. J. (1999). Treinamento de pais. In V. E. Caballo (Ed.), Manual de Técnicas de Terapia e Modificação do Comportamento (pp. 399422). São Paulo, SP: Santos.

Nix, R. L., Bierman, K. L., \& McMahon, R. J. (2009). How attendance and quality of participation affect treatment response to parent management training. The Conduct Problems Prevention Research Group US. Journal of Consulting and Clinical Psychology, 77(3), 429-438. doi:10.1037/a0015028

Pardo, M. B. L., \& Carvalho, M. M. S. B. (2011). Grupo de orientação de mães no contexto de uma clínica-escola. Paidéia (Ribeirão Preto), 21(48), 93-100. doi:10.1590/S0103$-863 X 2011000100011$

Patias, N. D., Siqueira, A. C., \& Dias, A. C. G. (2013). Práticas educativas e intervenção com pais: A educação como proteção ao desenvolvimento dos filhos. Advances in Health Psychology, 21(1), 29-40.

Patterson, G. R. (1986). Performance models for antisocial boys. American Psychologist, 41, 432444.

Pfiffner, L. J., Villodas, M., Kaiser, N., Rooney, M., \& McBurnett, K. (2013). Educational outcomes of a collaborative school-home behavioral intervention for ADHD. School Psychology Quarterly, 28(1), 25-36. doi:10.1037/spq0000016 
Posthumus, J. A., Raaijmakers M. A., Maassen, G. H., van Engeland, H., \& Matthys, W. (2012). Sustained effects of incredible years as a preventive intervention in preschool children with conduct problems. Journal of Abnormal Child Psychology, 40(4), 487-500. doi:10.1007/ s10802-011-9580-9

Power, T. J., Mautone, J. A., Soffer, S. L., Clarke, A. T., Marshall, S. A., Sharman, J., ...Jawad, A. F. (2012). A family-school intervention for children with ADHD: Results of a randomized clinical trial. Journal of Consulting and Clinical Psychology, 80(4), 611-623. doi:10.1037/a0028188

Reed, A., Snyder, J., Staats, S., Forgatch, M. S., DeGarmo, D. S., Patterson, G. R.; ...Schmidt, N. (2013). Duration and mutual entrainment of changes in parenting practices engendered by behavioral parent training targeting recently separated mothers. Journal of Family Psychology, 27(3), 343-354. doi:10.1037/a0032887

Rodrigo, M. J., Correa, A. D., Máiquez, M. L., Martín, J. C., \& Rodríguez, G. (2006). Family preservation services on the Canary Islands: Predictors of the efficacy of a parenting program for families at risk of social exclusion. European Psychologist, 11(1), 57-70. doi:10.1027/10169040.11.1.57

Sanders, M. R., Stallman, H. M., \& McHale, M. (2011). Workplace Triple P: A controlled evaluation of a parenting intervention for working parents. Journal of Family Psychology, 25(4), 581-590. doi:10.1037/a0024148

Santiago, C. D., Lennon, J. M., Fuller, A. K., Brewer, S. K., \& Kataoka, S. H. (2014). Examining the impact of a family treatment component for CBITS: When and for whom is it helpful? Journal of Family Psychology, 28(4), 560-570. doi:10.1037/a0037329

Smith, J. D., Dishion, T. J., Shaw, D. S., \& Wilson, M. N. (2013). Indirect effects of fidelity to the family check-up on changes in parenting and early childhood problem behaviors. Journal of Consulting and Clinical Psychology, 81(6), 962974. doi:10.1037/a0033950
Spoth, R., Randall, G. K., \& Shin, C. (2008). Increasing school success through partnershipbased family competency training: Experimental study of long-term outcomes. School Psychology Quarterly, 23(1), 70-89. doi:10.1037/10453830.23.1.70

Turner, K., \& Sanders, K. (2007). Family intervention in indigenous communities: Emergent issues in conducting outcome research. Australasian Psychiatry, 15, 39-43.

Van der Ende, P. C., van Busschbach, J. T., Nicholson, J., Korevaar, E. L., \& van Weeghel, J. (2014). Parenting and psychiatric rehabilitation: Can parents with severe mental illness benefit from a new approach? Psychiatric Rehabilitation Journal, 37(3), 201-208. doi:10.1037/ prj0000067

Velasquez, R., Souza, S. D., Adjuto, I., Muñoz, L. M., \& Silveira, J. C. C. (2010). O treinamento de pais e cuidadores: Ensinando a educar e promovendo a saúde mental. Revista Médica de Minas Gerais, 20(2), 182-188.

Weber, L. N. D., Brandenburg, O. J., \& Salvador, A. P. V. (2006). Programa de Qualidade na Interação Familiar (PQIF): Orientação e treinamento para pais. Psico, 37(2), 139-149.

Webster-Stratton, C. (1998). Preventing conduct problems in head start children: Strengthening parenting competencies. Journal of Consulting and Clinical Psychology, 66, 715-730.

Wong, J. J., Gonzales, N. A., Montaño, Z., Dumka, L., \& Millsap, R. E. (2014). Parenting intervention effects on parental depressive symptoms: Examining the role of parenting and child behavior. Journal of Family Psychology, 28(3), 267-277. doi:10.1037/a0036622 